University of Nebraska - Lincoln

DigitalCommons@University of Nebraska - Lincoln

May 2002

\title{
The Blazhko Effect of the RR Lyrae Star GV Andromedae
}

Kevin M. Lee

University of Nebraska-Lincoln, klee6@unl.edu

Edward G. Schmidt

University of Nebraska-Lincoln, eschmidt1@unl.edu

Shawn T. Langan

University of Nebraska - Lincoln, nuaur@hotmail.com

Follow this and additional works at: https://digitalcommons.unl.edu/physicsschmidt

Part of the Physics Commons

Lee, Kevin M.; Schmidt, Edward G.; and Langan, Shawn T., "The Blazhko Effect of the RR Lyrae Star GV Andromedae" (2002). Edward Schmidt Publications. 4.

https://digitalcommons.unl.edu/physicsschmidt/4

This Article is brought to you for free and open access by the Research Papers in Physics and Astronomy at DigitalCommons@University of Nebraska - Lincoln. It has been accepted for inclusion in Edward Schmidt Publications by an authorized administrator of DigitalCommons@University of Nebraska - Lincoln. 
Publications of the Astronomical Society of the Pacific, 114:546-550, 2002 May

(C) 2002. The Astronomical Society of the Pacific. All rights reserved. Printed in U.S.A.

\title{
The Blazhko Effect of the RR Lyrae Star GV Andromedae
}

\author{
Kevin M. Lee, Edward G. Schmidt, and Shawn T. Langan \\ Behlen Observatory, Department of Physics and Astronomy, University of Nebraska, Lincoln, NE 68588-0111; \\ klee6@unl.edu, eschmidt1@unl.edu, slangan1@bigred.unl.edu \\ Received 2001 June 27; accepted 2002 February 21
}

\begin{abstract}
We have obtained 462 new $V$ and $R$ observations of the RR Lyrae star GV Andromedae. Our data set is consistent with a primary period of 0.528092 days; however, the light curve of GV And cannot be well described by a single period. We conclude that GV And demonstrates the Blazhko effect with a Blazhko period of approximately 32 days.
\end{abstract}

\section{INTRODUCTION}

We have undertaken a survey of 107 Bailey type $a b \mathrm{RR}$ Lyrae stars in an effort to determine the frequency and characteristics of multiperiodic behavior. We observe the stars near the phase of maximum light and identify multiperiodicity in those stars for which the magnitude or phase of maximum does not repeat well from one cycle to another. At this time, observations have been finished for about two-thirds of the survey sample and at least 15 new multiperiodic stars have been identified. Complete light curves are then obtained for the multiperiodic stars as time allows, and these will be published as they are completed rather than await the conclusion of the survey. This is the fourth in a series of such papers that includes V442 Her (Schmidt \& Lee 2000), FM Per (Lee \& Schmidt 2001a), and DR And (Lee \& Schmidt 2001b).

A brief overview of multiperiodic behavior can be found in an earlier paper in this series (Lee \& Schmidt 2001a). Thorough coverage of the phenomena can found in Szeidl (1988) and Kovacs (2001).

The star GV And (GSC 02763-00880) is located at $\alpha=$ $23^{\mathrm{h}} 13^{\mathrm{m}} 12 \mathrm{~s} .5$ and $\delta=36^{\circ} 54^{\prime} 04^{\prime \prime}(\mathrm{J} 2000.0)$. Very little information exists on DR And in the literature. It is listed in the General Catalogue of Variable Stars (GCVS; Kholopov 1985, 1987 ) as having a primary period of 0.528092 days. No mention of any atypical behavior is made in the comments section. The only recently published observations were made by one of the authors (Schmidt 1991). Although Schmidt made only 15 observations of the star, he confirmed the star as a type RR $a b$ and found his data to be consistent with the GCVS period. He made no mention of any evidence for multiperiodicity.

\section{OBSERVATIONS}

We observed GV And 194 times between 1996 August 25 (JD 2,450,320) and 1997 February 15 (JD 2,450,494), an interval of 174 days. During a second observing run we obtained
268 points between 1997 May 15 (JD 2,450,583) and 1998 January 10 (JD 2,450,823), an interval of 240 days. The observing system has been described in an earlier paper in this series (Lee \& Schmidt 2001a). Because of the large number of points involved, the original photometric data will not be listed here. It is available in the IAU Archives of Unpublished Observations of Variable Stars in archive 327E. See Breger, Jaschek, \& Dubois (1990) for a description of the archives and how to access them.

The Behlen Observatory CCD photometry system and observational techniques were the same as described earlier (Schmidt 1991 and references therein). However, it should be emphasized that differential magnitudes were calculated for DR And with another star on the same CCD frames serving as a comparison star. Thus, accurate differential magnitudes were obtained even under inferior photometric conditions. On photometric nights standard stars (from Landolt 1983) were observed, and color terms for the transformation to the standard system were derived. Average values of these color terms over intervals of several months were then used to correct all nights within the interval. The resulting magnitudes of the comparison star (GSC 02763-00475) are $V=13.241 \pm 0.010$ and $R=$ $12.888 \pm 0.008$. Although the formal errors are small, they are based on only two photometric nights. Thus, the actual uncertainties might be as much as twice as large. Based on observations of other fields with multiple comparison stars of similar magnitudes, we estimate the quality of the GV And photometry between 15 and 20 mag in both $V$ and $R$.

It was not possible to observe a second comparison star of sufficient quality for good photometry on the same CCD frames. Thus, a third star in the same field as the comparison star served as a check star to verify the constancy in magnitude of the comparison star. The check star (GSC 0276300651) was observed at least once on every night of the second observing season, 16 nights spanning 240 days. The difference between the magnitudes of the check and com- 


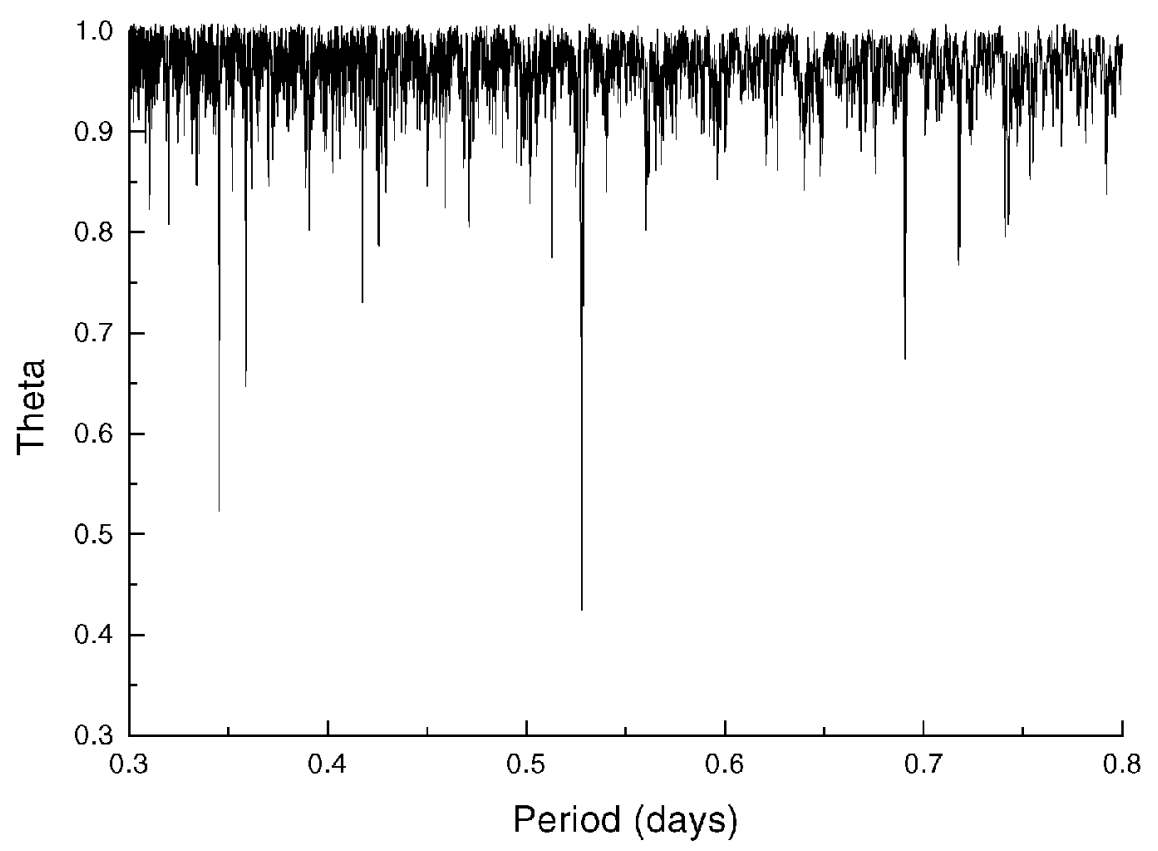

FIG. 1.-Power spectrum obtained from PDM analysis of the DR And $V$ data set.

parison stars had an rms of 35 mag. This is larger than the estimated error for the photometry of the variable star since the check star is much fainter.

\section{DATA ANALYSIS}

A period search was performed on the data using phase dispersion minimization (PDM; Stellingwerf 1978). In this method the data are binned according to a series of trial periods and a statistic $\theta$ is calculated for each trial period; $\theta$ is defined

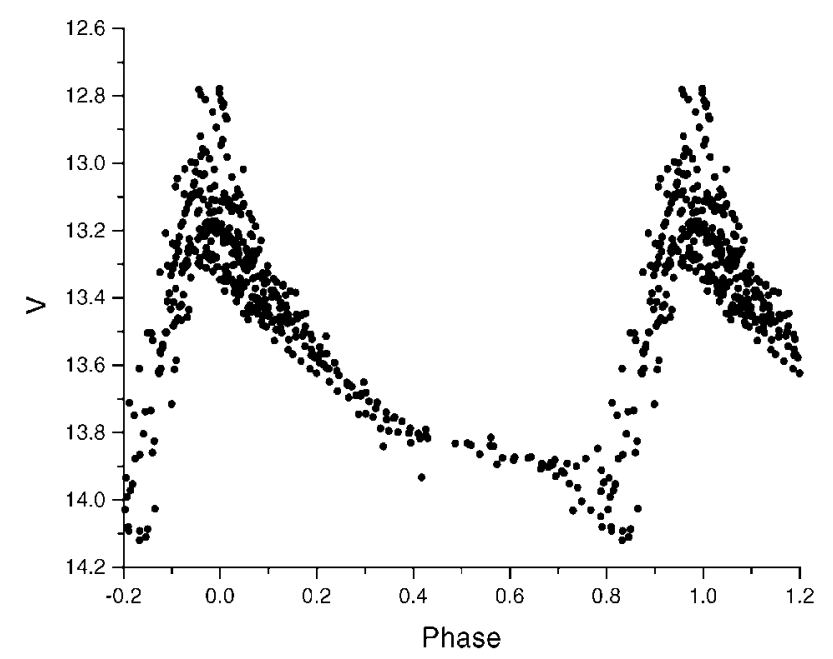

FIG. 2.-Light curve formed from the DR And $V$ data set where the phases have been determined by $\phi=(\mathrm{JD}-2,450,384.654) / 0.528092$. as the weighted average of all individual bin variances divided by the variance of the entire data set. The data were grouped into phase bins of $\left(N_{b}, N_{c}\right)=(5,2)$ in Stellingwerf's nomenclature. The PDM periodogram obtained from the $V$ data set is shown in Figure 1, where a periodic signal yields a significant minimum in $\Theta$. The most significant minimum corresponds to a period of 0.52812 days. The $R$ data set yielded an identical result.

However, the light curve obtained from the GCVS period of 0.528092 days was superior to that formed from the period of 0.52812 days in that it yielded considerably less scatter. Thus, we adopted the GCVS period, and the resulting light curve formed from our $V$ data is shown in Figure 2.

Visual inspection of this light curve is sufficient to convince one that DR And is multiperiodic and that the secondary periodicity has an amplitude of at least one-half a magnitude. Since the overall light-curve shape has the large amplitude and asymmetry typical of RR $a b$ stars, we began investigating double mode pulsation assuming that a 0.528092 day period was the fundamental period $P_{0}$. This would suggest a first overtone period of $P_{1}=0.396$ days. However, there is no local minimum in $\Theta$ near this value of period in Figure 1. Although unlikely, we investigated the possibility that the first overtone was the dominant mode of pulsation where $P_{1}=0.528092$ days and $P_{0}=0.704$ days. Although there is a local minimum in $\Theta$ near this value of period at $P=0.691$ days, we interpret this as twice the +1 cycle day $^{-1}$ alias of the fundamental period. However, neither of these results is surprising since the scatter in the light curve is much more pronounced near the phases of 


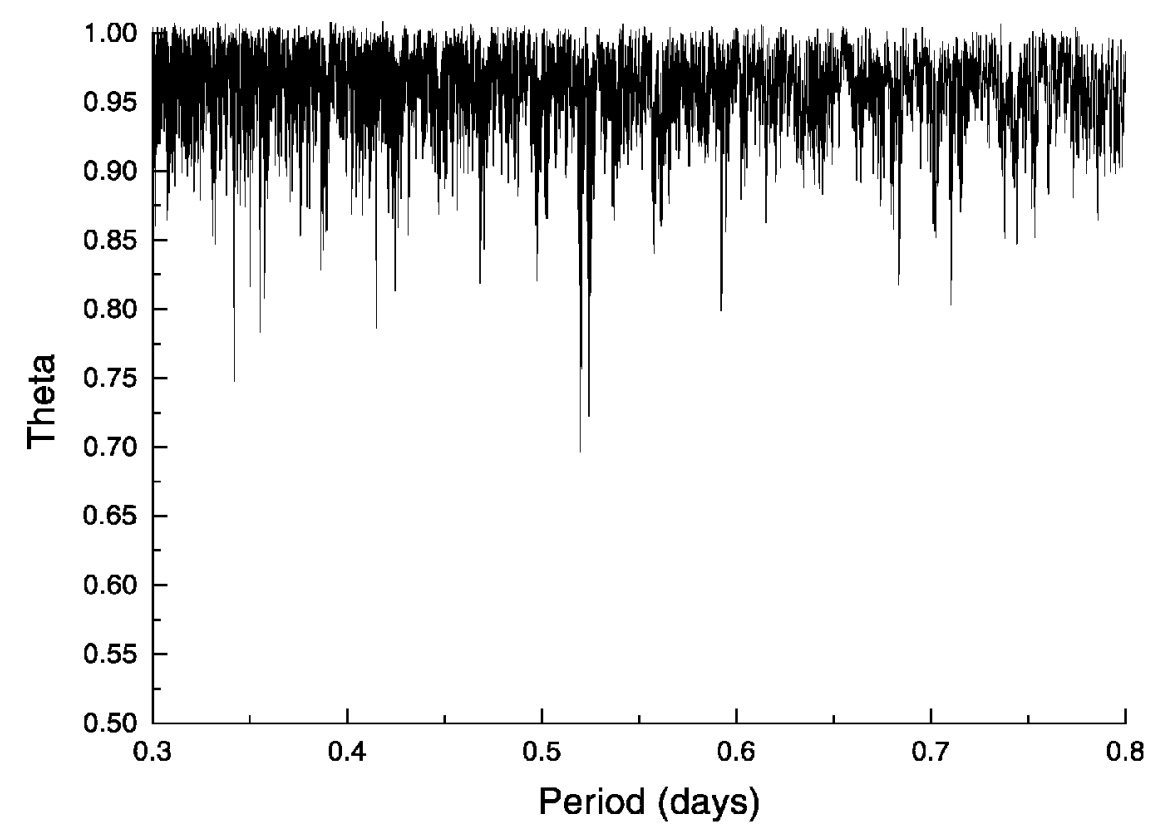

FIG. 3.-Periodogram obtained from running PDM on the prewhitened residuals of the $V$ data set.

maximum light, which is consistent with the Blazhko effect. This phenomenon has been observed in many Blazhko stars such as RV UMa (Kanyo 1976). However, this behavior is not seen in RR $d$ stars, where the secondary pulsation introduces scatter at all phases of the primary light cycle. A graphing of the light-curve points in chronological order allowed one to view the rise and fall of the magnitude of maximum light, which is also strongly suggestive of the Blazhko effect.

We looked for evidence supporting a Blazhko effect interpretation of the multiperiodicity using formal period search techniques. A mean light curve was formed using the LOWESS least-squares method (Cleveland 1981). The data were then prewhitened; the difference was calculated between the magnitude of each data point and the value of the mean curve at the same phase. It should be noted that very large residuals are obtained from data points on the ascending branch of the light curve. The residuals were searched for periodicity using the PDM routine, and the periodogram for the period range below 1 day is shown in Figure 3. One would expect the Blazhko period to manifest itself in the beating of the fundamental frequency against the Blazhko frequency. A minimum in $\theta$ is now observed at a period of 0.519635 days. This corresponds to a Blahzko period of

$$
\begin{aligned}
P_{B} & =1 /\left(\frac{1}{P_{\text {New }}}-\frac{1}{P_{0}}\right) \\
& =1 /\left(\frac{1}{0.519635 \text { days }}-\frac{1}{0.528092 \text { days }}\right)=32.4 \text { days. }
\end{aligned}
$$

There is another significant minimum at the nearby period of
0.524189 days, which would correspond to a Blahzko period of

$$
\begin{aligned}
P & =1 /\left(\frac{1}{P_{\text {New }}}-\frac{1}{P_{0}}\right) \\
& =1 /\left(\frac{1}{0.524189 \text { days }}-\frac{1}{0.528092 \text { days }}\right)=70.9 \text { days. }
\end{aligned}
$$

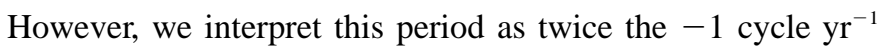
alias of the 32.4 day Blazhko period.

We next investigated the magnitudes of maximum light for help in discerning the Blazhko period. Table 1 lists the Julian Dates and magnitudes of GV And at maximum light obtained from the $V$ photometric data. Two different types of maximum light are identified: those for which the observations are sufficient in number and spacing so that the maximum is well defined (WD) and those for which the maximum may only be estimated (E). If not well defined, the magnitude of maximum light was estimated on a given night if there was a data point within 0.02 phase units of the phase of maximum light on the mean light curve. The magnitude of the point within 0.02 phase units was adopted as the maximum value. From the data in Table 1, one can calculate that the amplitude of the Blazhko effect is at least $0.49 \mathrm{mag}$ in $V$ and $0.43 \mathrm{mag}$ in $R$.

Figure 4 graphs the data from Table 1 (the magnitudes of maximum light vs. Julian Date). One can see from the variation in the magnitudes of maximum light that the Blazhko period must be on the order of tens of days. We define a "Blazhko curve" as a graph whose abscissa is the phase of magnitude 


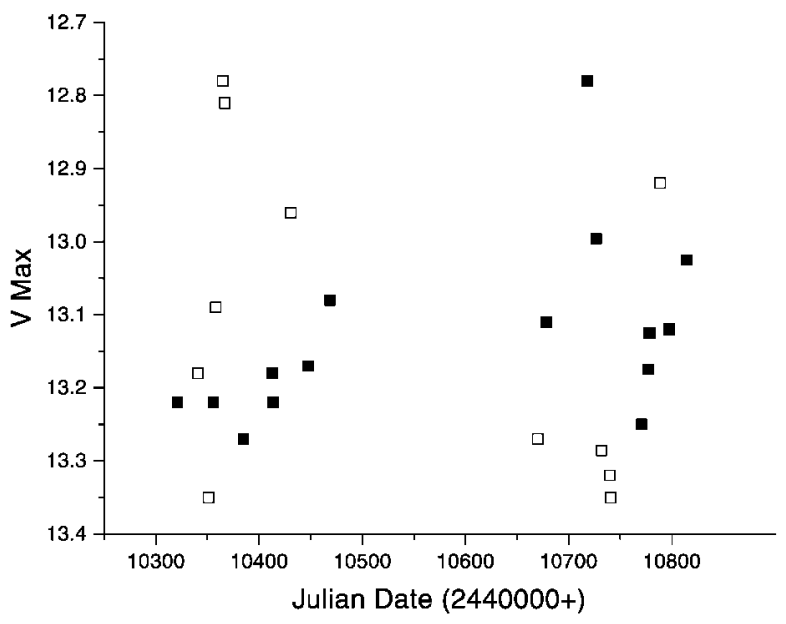

FIG. 4. $-V$ magnitudes of maximum light vs. Julian Date. The filled squares represent well-defined maxima, and the open squares represent estimated maxima.

light determined using the Blahzko period and whose ordinate is the magnitude of maximum light. Thus, the Blazhko curve shows the magnitude at maximum light in the primary pulsation cycle plotted as a function of phase in the Blazhko cycle. Figure 5 shows the Blazhko curve formed with phases determined by $\phi($ Blazhko $)=(\mathrm{JD}-2,450,320.77) / 32.4$. This Blazhko curve was superior to that formed from either the 35.5 day or the 70.9 day period, both of which had data points that did not fit the general trend of the other data.

We also applied PDM directly to the magnitudes of maxima in Table 1, and the resulting periodogram is shown in Figure 6. The most significant period was the 32.4 day period. The 35.5 day period is prominent as well. This approach of identifying the Blahzko period has the drawback of treating the well-defined maxima and estimated maxima with equal weight. When PDM is applied to only the well-defined maxima, similar results are obtained, although the periodogram has considerably more noise because of the smaller number of data points.

\section{CONCLUSIONS}

It is clear that GV And is a multiperiodic RR Lyrae star. The variation in the magnitude of maximum light is caused by the Blazhko effect, and it is consistent with a Blazhko period of approximately 32.4 days. Hopefully, as the number of known RR Lyrae stars exhibiting the Blazhko effect increases, a correlation may be identified between the Blazhko effect and some other stellar parameter that could shed light on the physical mechanism causing the effect.

The instrumentation used for the observations described in this paper was funded by the National Science Foundation (grant AST 85-04072). Support for publication expenses was

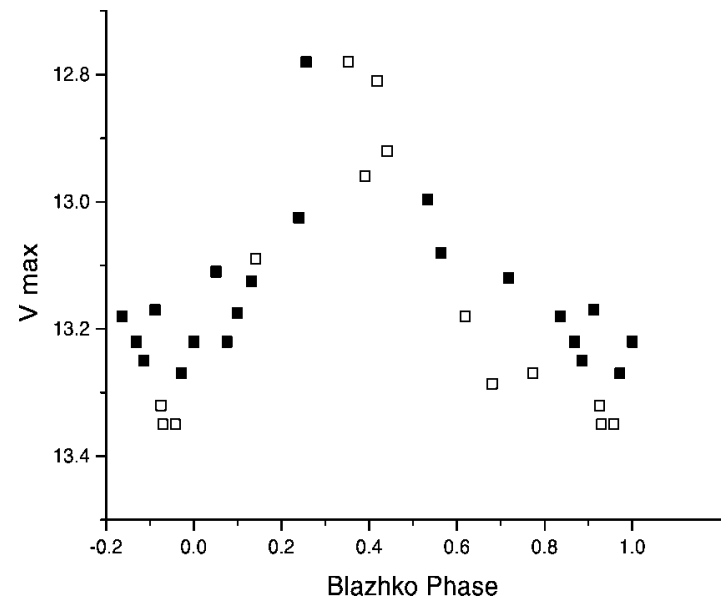

Fig. 5.-Blazhko curve formed from the $V$ magnitudes of maximum light. The Blazhko phases were determined from $\phi=(\mathrm{JD}-2,450,320.77) / 32.4$. The filled squares represent well-defined maxima, and the open squares represent estimated maxima.

provided by the American Astronomical Society through its small grants program. This research made use of the SIMBAD database operated at CDS, Strasbourg, France.

TABLE 1

Magnitudes of GV AND at Maximum Light

\begin{tabular}{|c|c|c|}
\hline $\begin{array}{l}\text { Heliocentric Julian Date } \\
\qquad(+2,440,000)\end{array}$ & $\begin{array}{c}V \text { Magnitude } \\
\text { (of Maximum Light) }\end{array}$ & $\begin{array}{l}\text { Definition of } \\
\text { Maximum }^{\mathrm{a}}\end{array}$ \\
\hline $10,320.77$ & 13.22 & WD \\
\hline $10,340.84$. & 13.18 & $\mathrm{E}$ \\
\hline $10,350.88 \ldots \ldots \ldots \ldots \ldots$ & 13.35 & $\mathrm{E}$ \\
\hline $10,355.62$. & 13.22 & WD \\
\hline $10,357.74$. & 13.09 & $\mathrm{E}$ \\
\hline $10,364.60$. & 12.78 & $\mathrm{E}$ \\
\hline $10,366.71 \ldots$ & 12.81 & $\mathrm{E}$ \\
\hline $10,384.655 \quad \ldots \ldots \ldots \ldots$ & 13.27 & WD \\
\hline $10,412.642 \ldots \ldots \ldots \ldots$ & 13.18 & WD \\
\hline $10,413.70 \ldots \ldots \ldots \ldots$ & 13.22 & WD \\
\hline $10,430.60 \ldots \ldots \ldots \ldots$ & 12.96 & E \\
\hline $10,447.505 \quad \ldots \ldots \ldots \ldots$ & 13.17 & WD \\
\hline $10,468.61 \ldots \ldots \ldots \ldots$ & 13.08 & WD \\
\hline $10,669.82 \ldots \ldots \ldots \ldots \ldots$ & 13.27 & $\mathrm{E}$ \\
\hline $10,678.798 \quad \ldots \ldots \ldots \ldots$ & 13.11 & WD \\
\hline $10,717.872 \quad \ldots \ldots \ldots \ldots$ & 12.78 & WD \\
\hline $10,726.84 \ldots \ldots \ldots \ldots \ldots$ & 12.996 & WD \\
\hline $10,731.62 \ldots \ldots \ldots \ldots \ldots$ & 13.286 & $\mathrm{E}$ \\
\hline $10,739.54$. & 13.32 & $\mathrm{E}$ \\
\hline $10,740.60 \ldots \ldots \ldots \ldots \ldots$ & 13.35 & $\mathrm{E}$ \\
\hline $10,770.67 \ldots \ldots \ldots \ldots$ & 13.25 & WD \\
\hline $10,777.556 \quad \ldots \ldots \ldots \ldots$ & 13.175 & WD \\
\hline $10,778.613 \ldots \ldots \ldots \ldots$ & 13.125 & WD \\
\hline $10,788.64 \ldots \ldots \ldots \ldots \ldots$ & 12.92 & $\mathrm{E}$ \\
\hline $10,797.609 \quad \ldots \ldots \ldots \ldots$ & 13.12 & WD \\
\hline $10,814.51 \ldots \ldots \ldots \ldots \ldots$ & 13.025 & WD \\
\hline
\end{tabular}

${ }^{\mathrm{a}} \mathrm{WD}=$ well defined; $\mathrm{E}=$ estimated. 


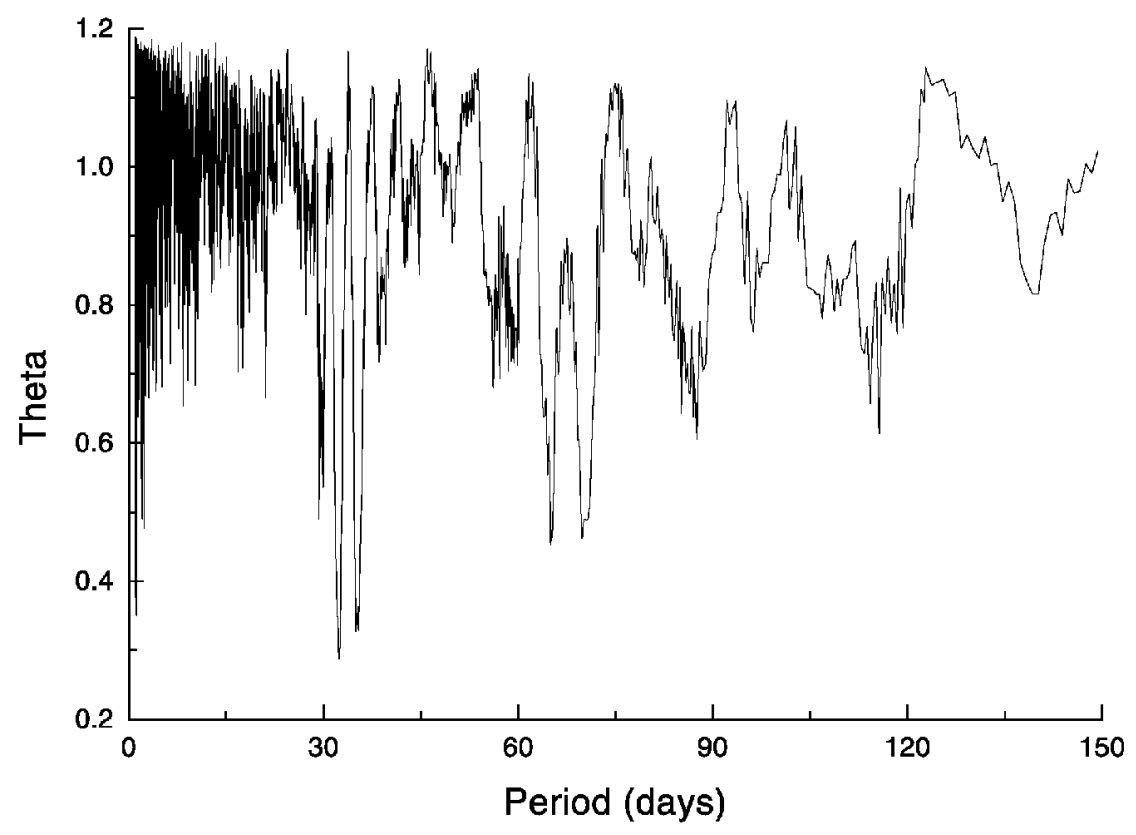

FIG. 6.-Periodogram obtained by running PDM on the $V$ magnitudes of maximum light listed in Table 1 .

\section{REFERENCES}

Breger, M., Jaschek, C., \& Dubois, P. 1990, Inf. Bull. Variable Stars, 3422

Cleveland, W. S. 1981, Am. Statistics, 35, 54

Kanyo, S. 1976, Commun. Konkoly Obs., 69, 1

Kholopov, P. N. 1985, General Catalogue of Variable Stars (4th ed.; Moscow: Nauka) 1987, General Catalogue of Variable Stars, Vol. 3 (4th ed.; Moscow: Nauka)

Kovacs, G. 2001, Stellar Pulsations-Nonlinear Studies (Dordrecht: Kluwer)
Landolt, A. U. 1983, AJ, 88, 439

Lee, K. M., \& Schmidt, E. G. 2001a, PASP, 113, 835 2001b, PASP 113, 1140

Schmidt, E. G. 1991, AJ, 102, 1766

Schmidt, E. G., \& Lee, K. M. 2000, PASP, 112, 1262

Stellingwerf, R. F. 1978, ApJ, 224, 953

Szeidl, B. 1988, in Multimode Stellar Pulsations, ed. G. Kovacs, L. Szabodos, \& B. Szeidl (Budapest: Konkoly Obs.), 45 